\title{
dite 1005
}
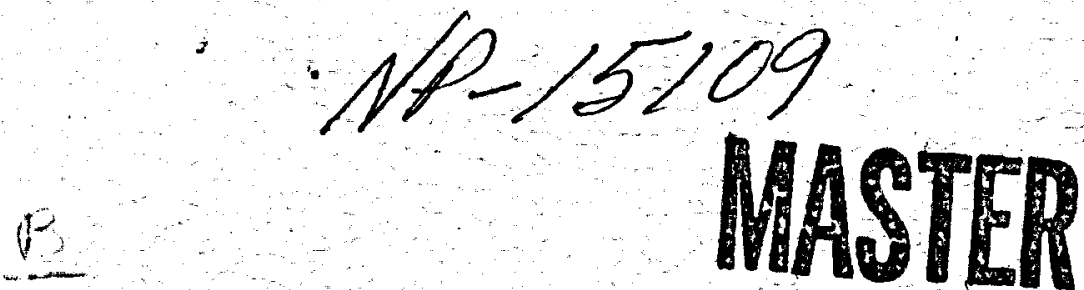

Phase-Space Integrals for Beta-Decay and Nuclear Matrix Elements ${ }^{t}$

\author{
John N. Bahcall
}

California Institute of Technology, Pasadena, California

Abstract: The use of phase-space integrals to determine nuclear matrix elements is discussed. The procedure for determining matrix elements is 11lustrated by analyzing the allowed and superallowed beta-decays of Isotopes with mass numbers in the range five to ten, utilizing the recent sumary of the properties of nuclel in this region complied by Ajzenberg-Selove and Lauritsen. Some 1 -values for other beta-decays

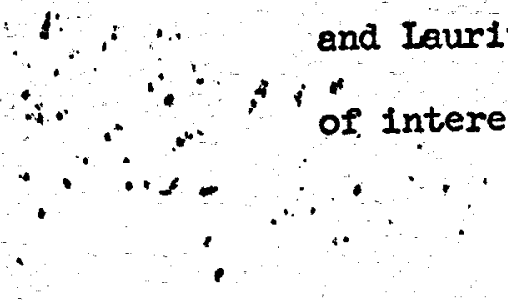

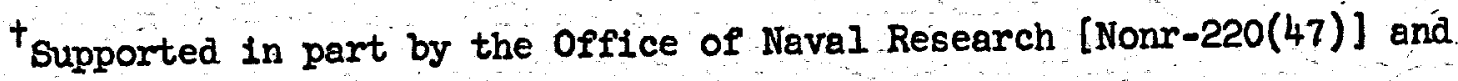
the National Aeroneutfes and Space Administration [NGR-05-002-028]. 


\section{DISCLAIMER}

This report was prepared as an account of work sponsored by an agency of the United States Government. Neither the United States Government nor any agency thereof, nor any of their employees, makes any warranty, express or implied, or assumes any legal liability or responsibility for the accuracy, completeness, or usefulness of any information, apparatus, product, or process disclosed, or represents that its use would not infringe privately owned rights. Reference herein to any specific commercial product, process, or service by trade name, trademark, manufacturer, or otherwise does not necessarily constitute or imply its endorsement, recommendation, or favoring by the United States Government or any agency thereof. The views and opinions of authors expressed herein do not necessarily state or refiect those of the United States Government or any agency thereof. 


\section{DISCLAIMER}

Portions of this document may be illegible in electronic image products. Images are produced from the best available original document. 


\section{Introduction}

Phase-space integrals for nuclear beta-decay, which are frequently called "f-values," are useful for two reasons. The phase-space integrals for a given transition can often be used in conjunction with the measured half-IIfe, $\tau_{\frac{1}{2}}$, and certain selection mules to determine uniquely the experimental nuclear matrix elements pertinent to the decay. The matrix elements thus obtained can then be compared with the predictions of theoretical models. Since the advent of the conserved vector current theory, several f-values have been determined very accurately $\left(\sim \frac{1}{2} \%\right)$ in order to test the prediction that pure Fermi transitions $\left(0^{ \pm} \rightarrow 0^{ \pm}\right)$have matrix elements that depend only on the isotopic spin quantum numbers of the initial and final nuclear states. One can also use the order of magnitude of the transition matrix elements, or equivalently the product $f \tau_{\frac{1}{2}}$, to help establish the spins and parities of the nuclear states that are involved. Transition matrix elements that pertain to different spin-parity changes, i.e., different degrees of forbiddenness, have values that characteristically differ by one or more orders of magnitude.

In this note, we are primarily concerned with showing how one can conveniently use phase-space integrals in conjunction with lifetime measurements and selection rules to determine nuclear matrix elements. We 1llustrate the procedure by analyzing the allowed and superallowed decays of isotopes with mass numbers in the range five to ten, utilizing the recent summary of the properties of nuclei in this region that has been compiled by Ajzenberg-Selove and Lauritsen 1). A future compllation by these authors will incorporate the results of a simflar analysis for radioactive nucle1 with mass numbers in the range ten to twenty. As a check on our numerical work, we have also calculated phase-space integrals for a number of standard decays that have previously been accurately studied. 


\section{Definition and Selection Rules}

The dimensionless phase-space factor, $f$, is defined for $\beta^{ \pm}$-emissions and for electron capture decays by the relation ${ }^{2)}$ :

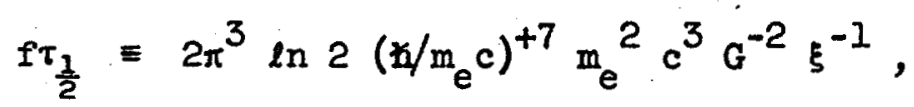

where $m_{e}, G$, and $\xi$ are, respectively, the electron's mass, the vector coupling constant of nuclear beta-decay, and a sum of squares of nuclear matrix elements. We first give explicit formulae for $f$ and then discuss the matrix elements that occur in $\xi$.

\subsection{Electron and Positron Fmissions}

For allowed beta-decays involving either electron or positron emission, one can show that ${ }^{2)}$ :

$$
\begin{aligned}
f & \equiv f\left( \pm z, W_{0}\right) \\
& =m_{e}^{-5} c^{-9} \int_{m_{e} c^{2}}^{W_{0}} d w( \pm Z, W) p W\left(W-W_{0}\right)^{+2},
\end{aligned}
$$

where $W_{0}$ is the difference between inftial and final nuclear energies (i.e., masses times $c^{+2}$ ) and $p, W$ are the emitted electron's momentum and energy respectively. The quantity $F( \pm Z, W)$ is just the ratio of the relativistic electron density at the nucleus to the density at infinity (hence $F \equiv I$ for $Z=0$ ). Of course, $f$ is proportional to the total phasespace available to the electron and neutrino in the final state. In our calculations, we have approximately taken account of the small effect due to the finite size of the nucleus by evaluating at the nuclear radius $R$ the expression for $F$ appropriate to a point nucleus. One thus 
obtains ${ }^{2)}$ :

$F( \pm Z, W)=2\left(1+\gamma_{0}\right)(2 \mathrm{pR} / \mathrm{z})^{-2\left(1-\gamma_{0}\right)} \mathrm{e}^{+\pi v}\left|\Gamma\left(\gamma_{0}+1 v\right)\right|^{+2} /\left[\Gamma\left(2 \gamma_{0}+1\right)\right]^{+2}$

where

$$
\gamma_{0} \equiv\left[1-(\alpha z)^{+2}\right]^{\frac{1}{2}}, \quad \alpha \equiv e^{2} / \text { hc, and } v= \pm \alpha \mathrm{zW} / \mathrm{cp} \text {. }
$$

The plus sign is to be used for electron emission and the minus sign for positron emission; the quantity $\mathrm{Z}$ is the charge of the final nucleus.

The above expression for $F$ should be corrected for the effect of screening by atomic electrons. This can be accomplished approximately ${ }^{3}$ ) by replacing $F( \pm Z, W)$ with

$$
F\left( \pm Z, W \mp v_{0}\right) \cdot\left[\frac{\left(W \mp v_{o}\right)^{2}-m_{e}^{2} e^{+4}}{w^{2}-m_{e}^{2} c^{+4}}\right]^{\frac{1}{2}}\left(\frac{w \mp v_{o}}{w}\right),
$$

where $\mathrm{v}_{0}$ is the shift in the potential energy at the nucleus caused by the screening. Durand ${ }^{4)}$ has recently shown that:

$$
v_{0} \approx 1.45 \alpha^{2}|z|^{+4 / 3} m_{e} c^{2}
$$

\subsection{Electron Cepture}

The appropriate phase-space factor for electron capture can be obtained from eq. (i) and the theory of allowed beta-decay. One finds:

$$
f_{\text {e.c. }}\left(z, w_{0}\right)=\pi^{2}\left(z / m_{e} c\right)^{+3} m_{e}^{-2} c^{-4} \Sigma_{n} a_{n s}\left|\Psi_{n s}(0)\right|^{+2} q_{n s}^{+2} \text {, }
$$

where $a_{n s}$ is the number of s-electrons in the inftial atom with principal quantum number $n, \Psi_{n s}(0)$ is the value of the electron's wave function. evaluated at the mucleus, and $q_{n s}$ is the energy of a neutrino emitted when 
an ns-electron is captured by the nucleus,

$$
q_{n s}=w_{0}+m_{e} c^{2}-b(n, s) \text {, }
$$

where $b(n, s)$ is the positive binding energy of an ns-electron in the initial atom. Accurate numerical expressions for $\Psi_{\mathrm{ns}}(0)$ are available for almost all cases of Interest from detailed self-consistent field calculations.

\subsection{Matrix Elements and Selection Rules}

The quantity $\xi$ that appears in eq. (1) is defined by the relation

$$
\xi \equiv\langle 1\rangle^{+2}+\left(C_{A} / C_{V}\right)^{+2}\langle\sigma\rangle^{+2}
$$

with

$$
\langle 1\rangle \equiv\left\langle I_{f}\left(M_{f}\right)\left|\sum_{i=1}^{A} \tau_{ \pm}(i)\right| I_{i}\left(M_{i}\right)\right\rangle
$$

and

$$
\langle\sigma\rangle \equiv\left\langle I_{f}\left(M_{f}\right)\left|\sum_{i=1}^{A} \sigma_{k}(1) \tau_{ \pm}(1)\right| I_{1}\left(M_{i}\right)\right\rangle\left\langle\left\langle I_{f}\left(M_{f}\right) I(k) \mid I_{i}\left(M_{i}\right)\right\rangle .\right.
$$

Here $I_{1}, M_{1}\left(I_{f}, M_{f}\right)$ are the total angular momentum and its projection along a definite axis of the nucleus in its initial (final) state; $\tau_{ \pm}$and $\sigma_{k}$ are ${ }^{5 \text { ), }}$ respectively, the familiar isotoplc spin raising and lowering operators and the Pauli spin matrices combined to form a spherical tensor of rank one. The ratio $\left(\mathrm{C}_{\mathrm{A}} / \mathrm{C}_{\mathrm{V}}\right)^{+2}$ is approximately:

$$
\left(C_{A} / C_{V}\right)^{+2}=1.40 \pm 0.05
$$

as we shall see later.

The matrix element $\langle 1\rangle$ vanishes unless $\Delta T=0^{+}, \Delta T=0$, and $\Delta T_{z}= \pm I$, 
where the superscript plus indicates no parity change and $T$ is the total Isotopic spin. Similarly, $\langle\sigma\rangle$ vanishes unless $\Delta I=0^{+}$or $\pm I^{+}$and $\Delta T=0, \pm I$, wth, of course, $\Delta T_{2}= \pm 1$.

The conserved vector current theory ${ }^{6)}$ implies that for transitions among members of an isotopic spin multiplet, i.e., superallowed decays:

$$
\langle 1\rangle^{2}=\left[T \mp T_{z}(1)\right]\left[T \pm T_{z}(i)+1\right]
$$

where the upper (lower) sign is to be used if the z-compound of the isotopic spin increases (decreases) in the transition. Here $T_{z}(i)$ is the $z$-component of the Isotopic spin in the initial state. Equation (1la) can also be written in the following more symetric form:

$$
\langle I\rangle^{2}=\left[T \mp T_{z}(1)\right]\left[T \pm T_{z}(f)\right]
$$

Equations ( $1 I^{2}$ ) and $(I I b)$ are of course valid for either convention [ $\tau_{z}$ (neutron) equals $+\frac{1}{2}$ or $\left.-\frac{1}{2}\right]$ regarding the sign of $T_{z}$.

\section{Numerical Results}

The expression for $f\left( \pm Z, w_{0}\right)$ that is obtained when eqs. (1)-(5) are combined has been programed for numerical calculations on an IBM 7094. For most of our computations we have used a nuclear radius $R$ equal to $1.3 \times 10^{-13} \mathrm{~A}^{1 / 3} \mathrm{~cm}$ and a screening potential given by eq. (5). For all cases with A less than ten that we have investigated, a change in the value of $R$ by $\pm 30 \%$ changes the resultant $f$-value by less than or of the order of a tenth of a percent. The results for higher $A$ are, of course, more sensitIve to the choice of $R$; for example, a $30 \%$ change in the nuclear radius results in a $1 \%$ change in the f-value for $\mathrm{v}^{46}$ and $\mathrm{Co}^{54}$. As one migit guess 
from eq. (4), the inclusion of electron screening results in a fractional change in the calculated $f$-values of the order of magnitude of $\mp 2 V_{o} /\left(w_{0}-m_{e} c^{2}\right)$, where the minus (plus) sign obtains for electrons (positrons). Equation (5) gives much too large a value for $\mathrm{V}_{0}$ in the special case of the decay of $\mathrm{H}^{3}$; fortunately, one can find $v_{0}$ exactly in this case with the aid of the known atomic wave function $\left[v_{0}\left(H^{3}\right)=e^{2} / a_{0} \approx 27 \mathrm{eV}\right]$. In addition to the effects we have included, there are ${ }^{7)}$ some other small corrections due to radiative effects, second forbidden contributions, overlap and exchange effects involving the atomic wave functions, and detalled averaging of the emitted electron's wave function over the nuclear surface. These corrections are small (typically $<1 \%)$ and can be ignored in all applications except those experiments designed to test the conserved vector current theory.

Some f-values calculated in the manner described above are given in column three of table 1; the cases shown were chosen because accurate hand calculations of the f-values for these decays already exist in the literature ${ }^{7-11)}$. For almost all cases, our results agree with the published values (without radiative corrections) to within a tenth of one percent. The only exceptions are the decay of $\mathrm{B}^{12}, \mathrm{~N}^{12}$, and $\mathrm{v}^{46}$. For both the $\mathrm{B}^{12}$ and $\mathrm{N}^{12}$ decays, our results appear to exceed the values of Fisher ${ }^{10)}$ by one percent; our results for these decays are in agreement with the values obtained by Kavanagh 11). our f-value for $v^{46}$ is three percent smaller than the f-value quoted by Jänecke ${ }^{8)}$.

In order to determine the uncertainty in $f$ that results from an experimental uncertainty in the energy difference $W_{0}$, it is convenient to introduce the quanitity

$$
\Delta \equiv I^{-1}\left(\frac{\partial f}{\partial W_{0}}\right)_{W_{0}}=W_{0}(\text { experimental })
$$


For a finite but small uncertainty, $\delta \mathrm{W}_{0}$, in the experimental energy, the fractional uncertainty in $f$ is then equal to $\Delta \times \delta W_{0}$. The calculated values of $\Delta$ are given in column four of table 1 in units of $\mathrm{MeV}^{-1}$.

The f-value for the decay of tritium is extremely sensitive to the energy, $W_{0}$, that is assumed. A change of $0.7 \mathrm{keV}$ in the assumed value of $W_{0}$ produces a ten percent change in the calculated f-value. Two apparently accurate experiments 9 ,12) have, in fact, yielded values of $\mathrm{w}_{0}$ that differ by $0.7 \mathrm{keV}$, Indicating that theorists should be cautious in making inferences that depend upon knowing the $f$-value of $\mathrm{H}^{3}$ to much better than $10 \%$.

\section{Nuclear Matrix Elements}

Equation (I) can be used to determine the nuclear matrix elements pertaining to a given transition if the $f$ and $\tau_{\frac{1}{2}}$ values (i.e., energy and lifetime) of the transition are known. For this purpose, it is convenient to eliminate the constants occurring in eq. (1) in favor of the $f \tau_{\frac{1}{2}}$-value of some standard transition whose matrix elements are well known from theoretical considerations and whose $f$-value and lifetime have been accurately measured. We adopt the $0^{14}+\mathrm{N}^{14 *}$ decay $\left(\langle 1\rangle^{2}=2,\langle\sigma\rangle^{2}=0\right)$, which has been extensively studied both tineoretically and experimentally. One can then rewrite eq. (I) as follows:

$$
\langle 1\rangle^{2}+\left(C_{A} / C_{V}\right)^{+2}(\sigma)^{2}=2\left(f \tau_{\frac{1}{2}}\right)_{0} 14 /\left(f \tau_{\frac{1}{2}}\right),
$$

where $\left.{ }^{13}\right)\left(f \tau_{\frac{1}{2}}\right)_{0} 14=(3.10 \pm 0.03) \times 10^{+3}$ secs.

The value of $\left(c_{A} / c_{V}\right)^{2}$ can be determined by inserting in eq. (13) the theoretical values $\left(\langle I\rangle^{2}=1,\langle\sigma\rangle^{2}=3\right.$ ) of the matrix elements for the neutron decay, the measured Iffetime of the neutron ${ }^{14}$ ), and the value of $I_{\text {neutron }}$ 
given in table 1. One thus finds the result, $\left(C_{A} / C_{V}\right)^{2}=1.40 \pm 0.05$, given in eq. (10), where the main uncertainty in $\left(C_{A} / C_{Y}\right)^{+2}$ arises from the uncertainty In the lifetime of the neutron.

Equations (10) and (13) can be combined to yleld a convenient equation for the nuclear matrix elements of an allowed or superallowed transition in terms of the experimentally determined $f \tau_{\frac{1}{2}}$-value. One finds:

$$
\langle 1\rangle^{2}+(1.40 \pm 0.05)\langle\sigma\rangle^{2}=(6.20 \pm 0.06) \times 10^{+3} /\left(f \tau_{\frac{1}{2}}\right) .
$$

The energies and iffetimes given in ref. (1) have been used to compute the most probable $f \tau_{\frac{1}{2}}$-values given in column five of table 2 for all allowed and superallowed decays of 1sotopes with mass numbers in the range five to ten. For $\mathrm{Be}^{7}$, which decays by electron capture, the values of $\left|\Psi_{n s}(0)\right|^{2}$ used in ref.' (15) were adopted. Errors in $f$ and $\tau_{\frac{1}{2}}$ are not explicitly indicated in table 2 since these may be readily inferred from the stated values of $\Delta$ and the indicated errors in the data ${ }^{l}$ ). Moreover, the errors in $f$ and $\tau_{\frac{1}{2}}$ are not as important for theoretical interpretations as the overall uncertainty in the nuclear matrix elements.

The nuclear matrix elements given in the last column of table 2 were calculated wth the aid of eq. (14), the selection rules given in section 2.3, and the computed $f \tau_{\frac{1}{2}}$-values. For all except the $\mathrm{Ii}^{9} \rightarrow \mathrm{Be}^{9}$ and $\mathrm{C}^{10} \rightarrow \mathrm{B}^{10^{* *}}$ decays, the largest uncertainty in the derived matrix elements is due to the uncertainty in $\left(c_{A} / c_{y}\right)^{+2}$, i.e., in the lifetime of the neutron. For both the $\mathrm{Id}^{9}+\mathrm{Be}^{9}$ and $\mathrm{C}^{10}+\mathrm{B}^{10 * *}$ decays, the largest uncertainty in the matrix elements is due to the lack of a precise measurement of the relevant branching ratios.

Some observations, not original, can be made on the basis of the matrix elements given in table 2. Notice first that matrix elements for superallowed 
decays are typically of the order of unity and matrix elements for allowed decays are typlcally of the order of one-tenth, although considerable dispersion from these values occurs. The fact that $\langle\sigma\rangle^{2}$ for $\mathrm{He}^{6}$ is less than the theoretical value of six which obtains for a ${ }_{S_{0}} \rightarrow{ }^{3} S_{1}$ transition suggests

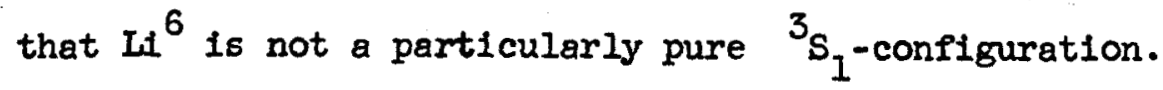

The most interesting entry in table 2 occurs in the last row, the decay of $\mathrm{C}^{10}$ to $\mathrm{B}^{10 * *}$. The value of $\langle 1\rangle^{2}$ predicted for this decay by the conserved vector current theory [see eq. (1I)] is two. The experimental value of $\langle 1\rangle^{2}$ differs from the predicted value by 0.8 , which is twice the quoted sum of errors. If one accepts the prediction of the conserved vector current theory and the estimate of the energy uncertainty ( $13 \mathrm{keV}$ ) given in ref. (1), then one can show that the branching ratio for the decay $\mathrm{C}^{10}+\mathrm{B}^{10^{* *}}$ should be $(2.2 \pm 0.15) \%$ instead of the quoted value of $(1.65 \pm 0.2) \%$. It would be use-ful to remeasure the branching ratio as well as the transition energy in order to remove the above mentioned discrepancy ${ }^{15)}$. In order to test the conserved vector current theory significantly with this decay, one would require a knowledge of the branching ratio and total I1fetime accurate to about $0.5 \%$ and $a$ knowledge of the transition energy accurate to about $1 \mathrm{keV}$. Since $\mathrm{c}^{10}$ is the lightest available 1sotope particlpating in a superallowed $0^{+}+0^{+}$ transition, an experiment which determined the branching ratio, total lifetime, and transition energy to the stated accuracy would also be useful in searching for a dependence upon mass number of such suggested interactions as a non-isotopic spin conserving part of the nuclear Hamiltonian.

It is a pleasure to thank Professor T. Lauritsen for supplying the relevant data for isotopes with mass numbers in the range five to ten. I am grateful to Dr. P. A. Seeger and B. A. Zimmerman for valuable assistance with the numerical calculations. 
Table 1

Some Calculated $f$-values

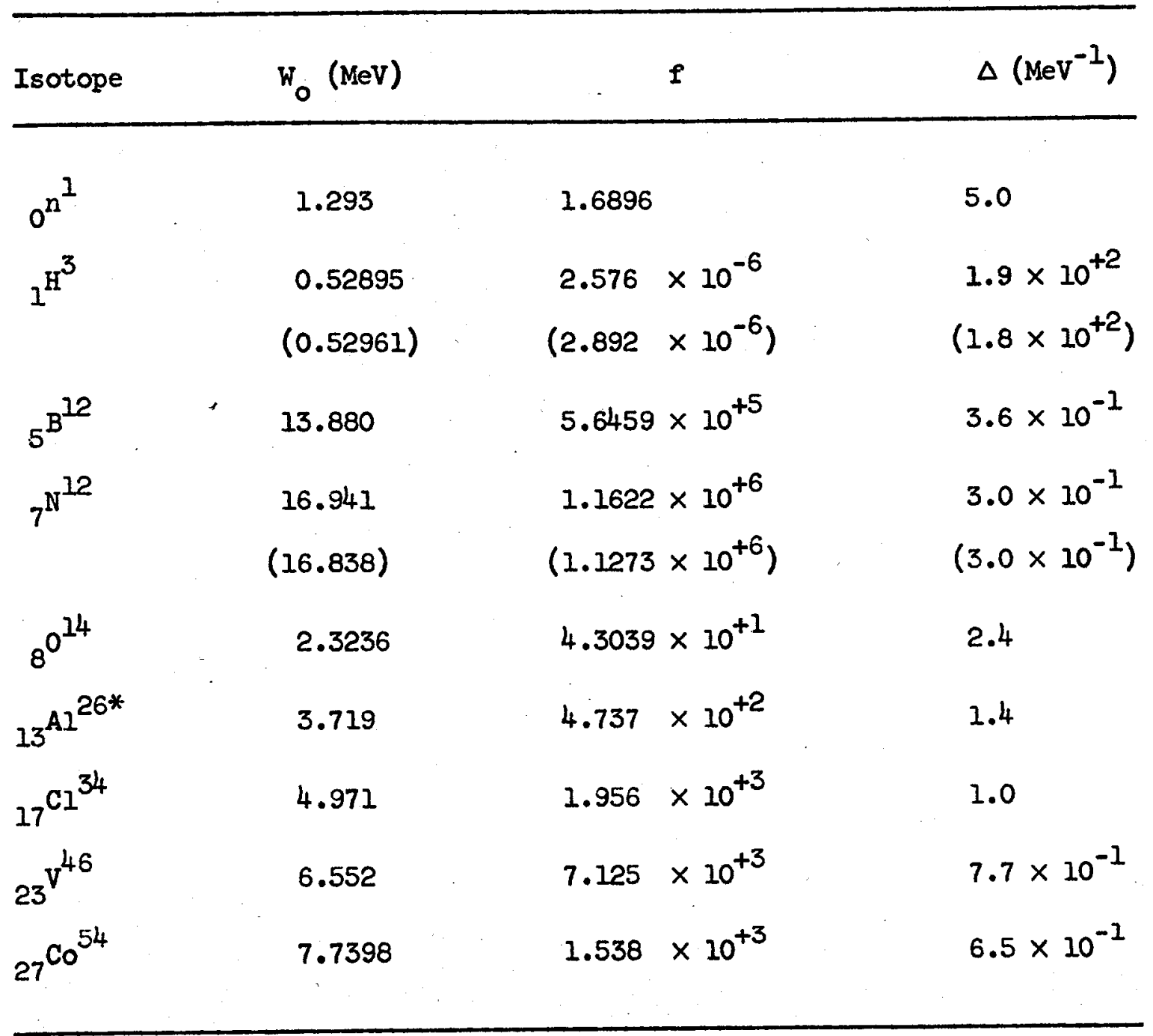


Table 2

The ft-values and nuclear matrix elements for allowed and superallowed

beta-decays of isotopes with mass numbers in the range five to ten.

\begin{tabular}{|c|c|c|c|c|c|c|}
\hline Isotope & $\mathrm{w}_{0}(\mathrm{MeV})$ & $f$ & $\Delta\left(\mathrm{MeV}^{-1}\right)$ & $P \tau_{\frac{1}{2}}(\mathrm{sec})$ & $\langle 1\rangle^{2}$ & $\langle\sigma\rangle^{2}$ \\
\hline $\mathrm{He}^{6}+\mathrm{II}^{6}$ & $4.0194 \pm 0.004$ & $1.00 \times 10^{+3}$ & 1.3 & $8.02 \times 10^{+2}$ & "0" & $5.6 \pm 0.2$ \\
\hline $\mathrm{Hi}^{8}+\mathrm{Be}^{8 *}$ & \pm 0.040 & $4.88 \times 10^{+5}$ & $3.7 \times 10^{-1}$ & $4.14 \times 10^{+5}$ & "0" & $(1.10 \pm 0.05) \times 10^{-2}$ \\
\hline $\mathrm{LH}^{9}+\mathrm{Be}^{9}$ & $14.126 \pm 0.040$ & $5.87 \times 10^{+5}$ & $3.5 \times 10^{-1}$ & $3.97 \times 10^{+5}$ & "0" & $(11 \pm 6) \times 10^{-3}$ \\
\hline $\mathrm{Li}^{9} \rightarrow \mathrm{Be}^{9 *}$ & \pm 0.040 & $2.28 \times 10^{+5}$ & $4.3 \times 10^{-1}$ & $5.13 \times 10^{+4}$ & "0" & $(8.8 \pm 0.4) \times 10^{-2}$ \\
\hline $\mathrm{Be}^{7} \rightarrow \mathrm{IH}^{7}$ & $0.8616 \pm 0.0003$ & $3.86 \times 10^{-4}$ & 2.3 & $2.00 \times 10^{+3}$ & "1" & $1.49 \pm 0.05$ \\
\hline $\mathrm{Be}^{7} \rightarrow \mathrm{Ii}^{7 *}$ & $0.3838 \pm 0.0004$ & $7.70 \times 10^{-5}$ & 5.2 & $3.45 \times 10^{+3}$ & "O" & $1.26 \pm 0.06$ \\
\hline$B^{8} \rightarrow B e^{8 *}$ & \pm 0.040 & $5.70 \times 10^{+5}$ & $3.4 \times 10^{-1}$ & $4.41 \times 10^{+5}$ & "0" & $(1.03 \pm 0.05) \times 10^{-2}$ \\
\hline $\mathrm{C}^{10}+\mathrm{B}^{10 *}$ & \pm 0.013 & $5.13 \times 10^{+1}$ & 2.3 & $1.01 \times 10^{+3}$ & "O" & $(4.5 \pm 0.2)$ \\
\hline $\mathrm{C}^{10}+\mathrm{B}^{10 * *}$ & $1.356 \pm 0.013$ & $1.90 \pm 0.12$ & 4.7 & $2,24 \times 10^{+3}$ & $\begin{array}{l}2.77 \\
\pm 0.4\end{array}$ & "O" \\
\hline
\end{tabular}


1) F. Ajzenberg-Selove and T. Lauritsen, Nuclear Physics $\sim$ (196)

2) E. J. Konopinsk1 and M. E. Rose, "The Theory of Nuclear B-Decay," in Alpha-, Beta- and Gamma-Ray Spectroscopy, edited by K. Siegbahn (North-Holland Publishing Co., Amsterdam, 1965) p. 1327. We use the notation of these authors; the reader is referred to their excellent review article for a concise sumary of the theory of nuclear beta-decay

3) M. E. Rose, Phys. Rev. 49 (1936) 727. See also C. Longmire and H. Brown, Phys. Rev. 75 (1949) 1102 and ref. 2)

4) I. Durand, III, Phys. Rev. 1355 (1964) B310

5). M. E. Rose, Elementary Theory of Angular Momentum (Wiley, New York, 1957)

6) R. P. Feynman and M. Gell-Mann, Phys. Rev. 109 (1958) 193

7) See, for example, L. Durand, III, L. F. Landovitz, and R. B. Marr, Phys. Rev. 230 (1963) 1188; J. N. Bahcall, Phys. Rev. 129 (1963) 2683; J. M. Freeman, J. H. Montague, G. Murray, R. E. White and W. E. Burcham, Phys. ' Letters 8) (1964) 215

8) J. Janecke, Phys. Letters 6 (1963) 69

9) I. M. Langer and R. J. D. Moffat, Phys. Rev. 88 (1952) 689

10) T. R. Fisher, Phys. Rev. 130 (1963) 2388

11) R. W. Kavanagh, Phys. Rev. 133 (1964) B1504

12) F. T. Porter, Phys. Rev. 115 (1959) 450

13) R. K. Bardin, C. A. Barnes, W. A. Fowler and P. A. Seeger, Phys. Rev. 127 (1962) 583

14) A. Sosnovski1, P. Spivak, Yu. Frokoviev, I. Kutikov and Yu. Dobrynin, JEIP (Soviet Physics) 35 (1959) 739

15) J. N. Bahcall, Phys. Rev. 128 (1962) 1297

16) The existence of this discrepancy has been recognized by a number of people; see particularly, F. J. Bartis, Phys. Rev. $\underset{\sim}{132}$ (1963) 1763 